\title{
Neu, int-1, int-2 et cancer du sein
}

\section{Les nouvelles \\ de ce numéro ont été préparées par Jean-Claude Dreyfus Jean-Pierre Grünfeld Axel Kahn Marc Peschanski}

1. Van de Vijver MJ, Peterse JL, Mooi WJ, et al. Neu protein overexpression in breast cancer: association with Comedo-type ductal carcinoma in situ and limited pronostic value in stage II breast cancer. $N$ Engl J Med 1988 ; 319: 1239-45.

2. Muller WJ, Sinn E, Pattengale PK, Wallace $R$, Leder $P$. Single-step induction of mammary adenocarcinoma in transgenic mice bearing the activated c-neu oncogene. Cell 1988; 54 : 105-15.

3. Tsukamoto AS, Grosschedl R, Guzman RC, Parlow T, Varmus HE. Expression of the int-l gene in transgenic mice is associated with mammary gland hyperplasia and adenocarcinoma in male and female mice. Cell $1988 ; 55: 619-25$.

4. Mansour SL, Thomas KR, Capecchi MR. Description of the proto-oncogene int-2 in mouse embryo-derived stem cells: a general strategy for targeting mutations to non-selectable genes. Nature $1988 ; 336: 348-52$.

$\mathrm{m} / \mathrm{s} n^{\circ} 2$ vol. 5 , février 89
Nos lecteurs savent tout de la controverse scientifique existant quant à la valeur pronostique de la modification d'oncogènes dans le cancer du sein $\left(\mathrm{m} / \mathrm{s} n^{\circ} 8\right.$, vol. $\left.4, p .524,528\right)$. Une nouvelle équipe, néerlandaise, utilisant cette fois-ci la détection immunologique de la protéine Neu, ne trouve pas de signification pronostique à l'hyper-expression de l'oncogène neu dans le cancer mammaire; en revanche, la synthèse de Neu paraît être un bon marqueur d'un sous-type de cancer mammaire, indépendamment de son stade évolutif [1]. Il ne fait cependant aucun doute que neu activé est un oncogène fort ! L'activation consiste en une mutation ponctuelle de la partie du gène codant pour la région transmembranaire de la protéine Neu, un probable récepteur de membrane similaire au récepteur d'EGF. Des souris transgéniques exprimant l'oncogène neu dont l'expression a été ciblée vers la glande mammaire par l'utilisation des régions de contrôle du virus MMTV (mouse mammary tumor virus) développent des tumeurs massives de la glande, très probablement polyclonales, ce qui signifie que neu activé est, pour le tissu mammaire, un oncogène «complet » suffisant à lui seul pour cancériser la cellule [2]. L'oncogène neu est également exprimé dans les glandes salivaires des souris transgéniques, sans provoquer cependant autre chose qu'une hyperplasie de ces tissus.

Un autre oncogène impliqué, au moins chez la souris, dans la carcinogenèse mammaire est int-1, au niveau duquel s'insère le virus MMTV lorsqu'il provoque le développement d'une tumeur. Des souris transgéniques exprimant une construction dans laquelle l'oncogène int-1 est placé sous le contrôle du LTR (long terminal repeat) de MMTV développent également des tumeurs mammaires chez les femelles et chez les mâles. De plus, quelques adénocarcinomes des glandes salivaires sont ici observés [3].

La progression tumorale est, dans le cas de int-1, plus « classique », puisque les tumeurs apparaissent être probablement clonales, nécessitant donc l'intervention d'un événement surajouté à l'expression du transgène. Des études ultérieures diront si, de la même manière, les autres gènes à proximité desquels le virus MMTV peut s'intégrer (int-2, int-3...) sont en effet des oncogènes. Les résultats concernant int-2 pourront revêtir une particulière signification en pathologie humaine, puisque cet oncogène semble fréquemment remanié dans le cancer du sein $\left(\mathrm{m} / \mathrm{s} n^{o} 8\right.$, vol. 4, p. 528). Int-2 est un analogue du gène codant pour FGF (fibroblast growth factor) et, comme ce dernier, pourrait être un gène jouant un rôle important dans l'embryogenèse précoce [4]. La récente réussite de l'équipe de Mario R. Capecchi (Howard Hughes Institute, Salt Lake City, UT, USA) qui a pu réaliser une mutagenèse insertionnelle de int-2 par recombinaison homologue (nous reparlerons en détail de ce problème ultérieurement) permettra peut-être de préciser le rôle de cet oncogène dans le développement. 